\title{
Results from Investigating Powered Wheelchair Users Learning to Drive with Varying Levels of Sensor Support
}

\author{
David Adrian Sanders \\ School of Engineering \\ University of Portsmouth \\ Portsmouth, UK \\ david.sanders@port.ac.uk \\ Alexander Gegov \\ School of computing \\ University of Portsmouth \\ Portsmouth, UK \\ alexander.gegov@port.ac.uk
}

\author{
Benjamin John Sanders \\ Peter Symonds College \\ Owens Road \\ Winchester, UK \\ ben.sanders@ @erg.org.uk
}

\author{
David Ndzi \\ School of Engineering \\ University of Portsmouth \\ Portsmouth, UK \\ david.ndzi@port.ac.uk
}

\begin{abstract}
This paper presents some results from investigating wheelchair driver learning when using different amounts of sensor support. A quantitative and qualitative empirical evaluation is made about the effect of using intelligent sensor support while a participant learns to operate a wheelchair. Reliance on teaching processes was recorded while a variety of levels of support were provided. Results are presented.
\end{abstract}

Keywords-Learning; powered wheelchair; ultrasonic; sensor

\section{INTRODUCTION}

The way that powered wheelchair (PW) users adapt their actions when presented with various distinct levels of support from a sensor system is explored to assess the outcome of giving assistance as users learn how to drive a PW.

The appropriate level of automated assistance depends on many things. This research explored the way that wheelchair drivers ought to be taught if different levels of support could be provided. Some research had suggested that sometimes, an experienced operator trained to operate a $\mathrm{PW}$ base without sensors might complete tasks even more efficiently with sensors to help them [1]-[3]. This paper considers whether a skilled operator trained with a sensor assisting them, could then work well in situations without having any sensors provided. Changes in the behaviour of participants were monitored while they were given varying amounts of support. In this case, they changed their behaviour in different environments or in new circumstances.

In traditional research to investigate using various levels of support, the participants have usually been experts with predeveloped skills. Acquiring skill has usually been ignored [4].

Driving a powered wheelchair fitted with sensors was used as a realistic case where humans interact with computers. The results from the experiments are presented.
Some investigations that used industrial robots are described in [5], [6]. In the work described in this paper, constraint-based support that used virtual force feedback was used [4]. Force feedback represented the closeness to a wall or obstacle and this was similar to work by Volpe who represented force on a slave arm back at a joystick [7]. Constraint-based support provided a force back at the controller in order to evade collisions and direct an operator in a safer direction.

The PW system is described in [3]. Part II of this paper describes the experiments and presents some results. Part III is some discussion and conclusions.

\section{EXPERIMENTATION}

A choice of three different levels of support could be provided to a wheelchair driver:

Lev0: Ultrasonic were turned off so that there was not any force feedback. The wheelchair driver could steer their wheelchair with no interference from the sensors.

The operator had the greatest autonomy with Lev0 but the likelihood of crashing was greatest.

Lev1: Sensors were engaged and the joystick received force feedback when the PW was driven close to objects in its path. The size of repelling force was increased as distance to an object reduced.

Lev2: Sensors were engaged and the PW was steered automatically away from objects to avoid collisions. A human wheelchair user did not sense any resistive force if he/she moved the PW in the direction of any obstacles, the PW was safely steered.

Lev3: Both of Lev1 and Lev2 were delivered at the same time. Operators would be most constrained in this case. 
If they applied more effort to their joystick then wheelchair drivers could make their PW move in opposition to the deflecting force.

Number of collisions (C) and average completion time (T) were employed as performance measures.

\section{A. Initial Tests}

The initial tests compared learning in Lev0 (with sensors off) and Lev3 (with sensors on, a repulsive force and automatic avoidance to steer the PW safely around obstacles). Subsequent tests compared learning at Lev0, Lev1 and Lev2 in different environments.

Participants drove a Bobcat II PW [3] through one of four different courses.

Courses were different lengths and contained different numbers of obstacles.

Participants used a joystick connected to the PW to drive the PW through each course while attempting to avoid obstacles.

A Forcefeedback Pro joystick from Microsoft was used to steer the PW. It had force feedback built in and had been designed and used for games. It was unable to apply force quantitatively, but it had already been successfully used as a force feedback mechanism in some experimental studies at Tohoku University in Japan [4] and so was selected for this work.

For Lev3 tests, the resistive force grew bigger when a participant drove their PW close to an obstacle. So that the assistance provided was more restricted than Lev1 or Lev2 alone.

Volunteers were sixty staff and students (with no prior experience). They were separated in to two groups (A \& B). They were further sub-divided between the four courses used for testing. There were about eight participants within each sub-group.

Participants were shown their PW route and obstacles along the route. Then they carried out the driving task ten times with sensors and 10 times without. Then, the groups redid each test but with a different level setting. A second set of tests examined the execution of the task when a PW driver was confronted a new set of supporting conditions after developing their PW driving skill.

Subjective information about preference was collected using a simple questionnaire. The questions were:

"Do you prefer Lev0 or Lev3 support?"

"Is it easier to drive with Lev0 or Lev3?"

Answers are recorded in Table 1.

\section{B. Results from Initial Testing}

Average completion times in seconds for A and B for each attempt are shown in Fig. 1 and 2 for the four courses.

Tests 1 to 10 correspond to the initial tests (A with Lev3 support and B with Lev0) and 11 to 20 show the later tests (B with Lev3 support and A with Lev0).
TABLE I. SUBJECTIVE APPRAISAL (OBTAINED By QUESTIONNAIRE)

\begin{tabular}{|l|l|l|l|l|l|}
\hline & $\begin{array}{l}\text { Strongl } \\
\mathbf{y} \\
\text { prefer } \\
\text { Level 0 }\end{array}$ & $\begin{array}{l}\text { Prefer } \\
\text { Level } \\
\text { 0 }\end{array}$ & $\begin{array}{l}\text { Undecid } \\
\text { ed }\end{array}$ & $\begin{array}{l}\text { Prefer } \\
\text { Level } \\
3\end{array}$ & $\begin{array}{l}\text { Strongly } \\
\text { prefer } \\
\text { Level 3 }\end{array}$ \\
\hline Preference & 13 & 15 & 17 & 12 & 3 \\
\hline $\begin{array}{l}\text { How } \\
\text { Easy? }\end{array}$ & 14 & 17 & 8 & 15 & 6 \\
\hline
\end{tabular}

Average completion time



Attempts made at completing the course ->

Fig. 1. Average completion time $\mathrm{T}$ for Groups $\mathrm{A}$ and $\mathrm{B}$ over four dissimilar courses. Lev3 support first, then Lev0.

Average completion time

$\mathrm{T}$



$\begin{array}{llllllllll}1 & 2 & 3 & 4 & 5 & 6 & 7 & 8 & 9 & 101112131415161718192 C\end{array}$

Attempts made at completing the course ->

Fig. 2. Average completion time $\mathrm{T}$ for Groups $\mathrm{A}$ and $\mathrm{B}$ over four dissimilar courses. Lev0 first then Lev3. 
Fig. 1 and 2 shows that volunteer Drivers in A (with force feedback) achieved a learning equilibrium sooner and more steadily than B. This suggests a helpful effect from using Lev3 during the initial learning when skill was being developed. Drivers in A, also performed better during the second part of the tests, with Lev0 support. Skill acquisition was accelerated using Lev3 support.

Drivers may have attained general skill that could then be applied to new conditions (in this case operating the wheelchair without any support). Drivers in B did not show improvement in the second half of the trials (in terms of time taken and stability). That behaviour might be because skill developed with Lev0 support dos not transfer so easily to new situations or conditions.

Table 1 shows the results from subjective appraisal (acquired from the questionnaires). Participants said it was simpler to drive without any assistance from the sensor systems (Lev0) but that disagreed with the more objective results. In addition, the questionnaire answers suggested that participants did not like being assisted.

\section{Discussion of Initial Testing}

The initial tests suggested PW drivers trained with sensors helping them could deal with new situations when they were not being assisted. That is when the sensors were switched off or removed.

Having some support from sensors during the learning phases appears to have had a significant effect on future working when participants completed driving tasks without any sensor assistance.

It is possible that participants may have developed an understanding of the systems and of driving the PW along a path during sequences of tests. More tests are needed to validate the results to confirm that a developed driving skill can transfer to a different working condition; one without any assistance from sensors.

Results do agree with results presented by Chikura [4] but the performance of participants faced with different tasks must be examined further to confirm and generalize the results.

Results suggest that PW drivers trained without any assistance from sensors did not function any better than drivers being assisted by sensors.

Results also indicated that participants that were taught without any assistance did not appear to learn steadily when compared with participants without previous experience. It is possible that skills obtained while driving without sensor assistance had an adverse effect on driving performance when assistance was added later. That is important for training procedures for intelligent systems.

Informal discussion revealed that was partially as the teloperators felt their freedom of decision making and movement was being constrained

That contradiction between objective execution and the self-biased comments indicated that decisions about training based on student preference could lead to poorer implementation.

\section{Second Set of Tests}

The second set of experiments investigated the way that participants adapted their conduct if work conditions changed. Participants used different levels of sensor assistance.

A different route was utilized for the second set of tests. It was more complicated and longer. Obstacles could be moved to easily create three discrete (but related) test runs using the same general route.

The second set of tests were to investigate differences in the behaviour of the participants if different levels of sensor support were provided and working conditions were changed.

Driving routes were carried out six times. Then routes were modified by moving obstacles and the participants drove the second route six times. Then routes were modified again by moving obstacles to new positions for a third set of six tests.

Each participant completed each test with the same level settings for each of their attempts.

Twelve new participants were separated into groups: Group X, Group Y, Group Z. X undertook tasks at Lev0 (without assistance), $Y$ at Lev1 (sensors generating a repulsion force) and $\mathrm{Z}$ at Lev2 (automatic steering around obstacles).

The number of collisions and the time taken were logged.

\section{E. Results from Second Set of Tests}

Fig. 3 to 5 show the time taken in seconds for Groups X, Y and $\mathrm{Z}$.

Average completion time

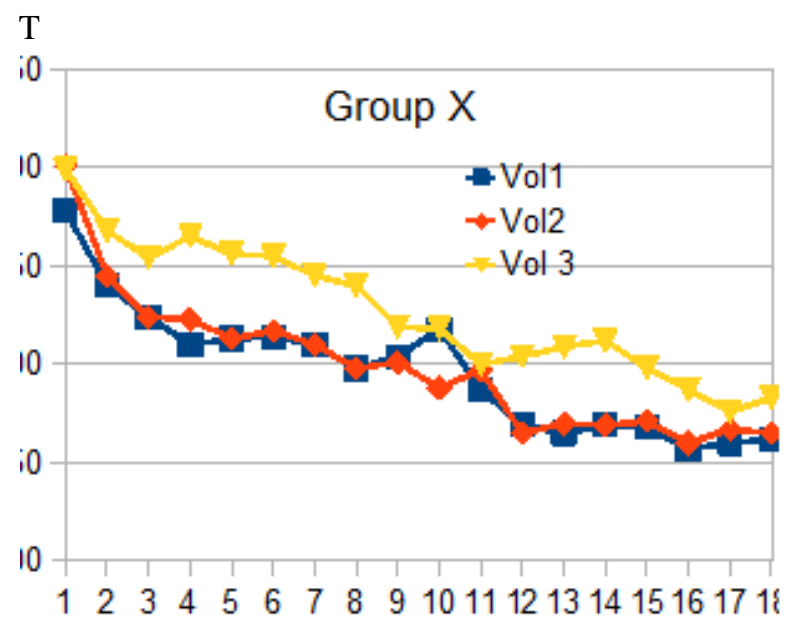

Attempts made at completing the course -> Fig.3. Lerv0 - Ultrasonic sensor system switched off.

Lev3 support resulted in the least collisions but completion times became worse compared with Lev0 support.

Differences in the completion times when the layout of the course (position of obstacles, etc.) was adjusted was assessed. Completion time tended to increase for Lev1 and Lev2, while completion time did not change for Lev0. 
Average completion time

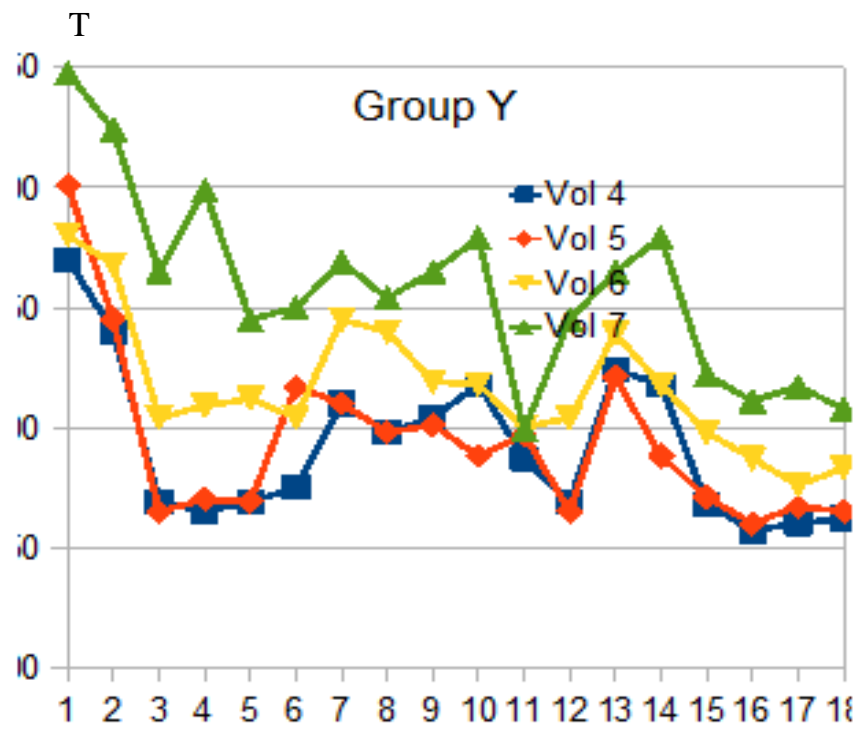

Attempts made at completing the course ->

Fig. 4. Lev1 - Ultrasonic sensors providing a repulsive force.

Average completion time

$\mathrm{T}$

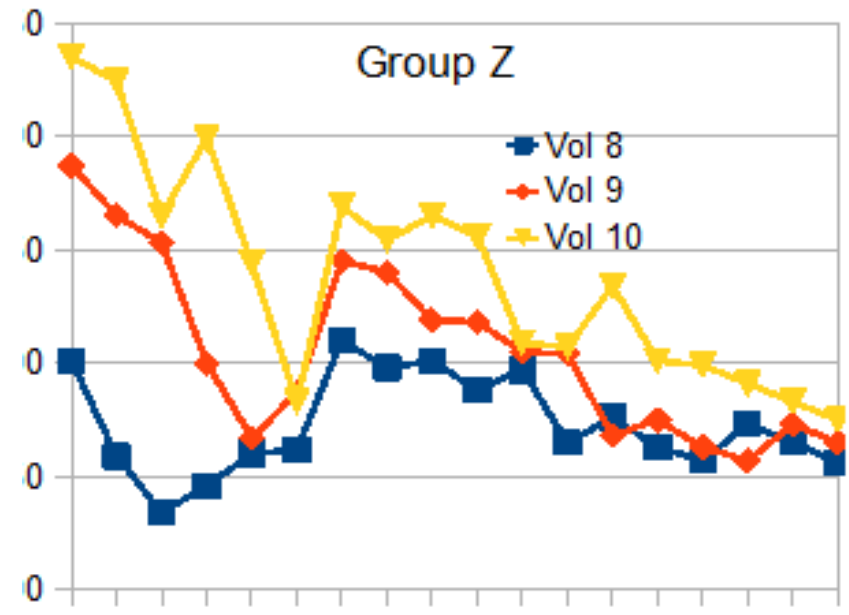

$\begin{array}{llllllllll}1 & 2 & 3 & 4 & 5 & 6 & 7 & 8 & 9 & 101112131415161718\end{array}$

Attempts made at completing the course ->

Fig. 5. Lev2 - System automatically steered the PW away from obstacles.

This could suggest that skills learned without sensors supporting the operator could be more generic when compared to skills learned with sensor support.

The number of collisions is shown in Fig. 6 for each volunteer.

There appeared to be differences in behaviour and adaptation when different levels of assistance were provided and when participants come upon different working conditions.

$\mathrm{T}$ showed different behaviour for each level when the course was changed.

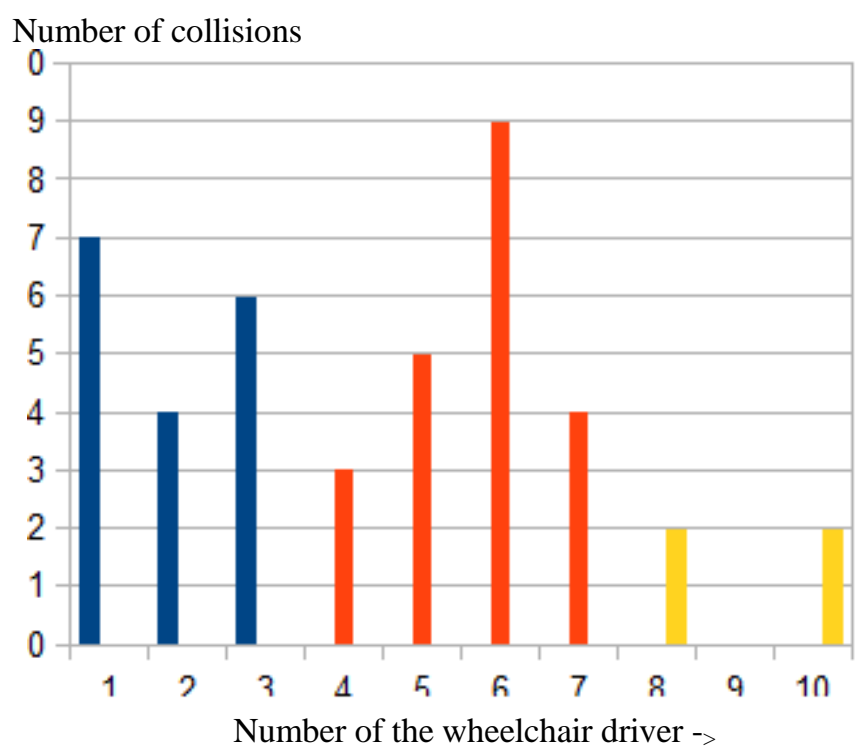

Fig. 6. Number of collisions for each wheelchair driver.

\section{DISCUSSION AND CONCLUSIONS}

The way that participants adapted their behaviour was investigated when different levels of assistance were provided to decide if using sensors to assist during learning had any effect, and if so then what effect did they have?

A positive effect was observed when learning with assistance from sensors. A harmful effect was observed when learning without any assistance from the sensors but then using sensors later to assist participants with their driving. Driving at Lev2 decreased the number of collisions in a variety of situations and arrangements of obstacles.

A larger number of participants could have made the results more significant. Additionally, the second set of tests contradicted some results obtained during initial tests.

The first set of tests suggested that support was helpful during learning but that was not seen in the second set of results. More tests are needed to focus on individual performance to acquire more unambiguous results. That said, the results have offered some insights about driver behaviour when learning with different levels of assistance provided by sensor systems.

While this work has put forward a thesis that using sensors during training is effective, some research has suggested that if a driver is proficient then they function better without any help in open and safe conditions. In all situations though, assistance form sensors become more useful as environments become more complicated.

Work is now investigating mixing other AI tools [8]-[17] to use specific tools where they can have most effect.

\section{REFERENCES}

[1] Sanders DA; Graham-Jones J and Gegov A. Improving ability of teleoperators to complete progressively more difficult mobile robot paths using simple expert systems and ultrasonic sensors. Industrial Robot Volume: 37, Issue: 5, Pages: 431-440. 2010 
[2] Sanders, D. A., Bausch, N. C. \& Ndzi, D. L Changes to user learning behaviour of powered wheelchair drivers depending on the level of sensor support. IEEE Proc' of International Conference Health Informatics and Medical Systems HIMS'15. Arabnia, H. R. \& Deligiannidis, L. (eds.). Published by CSREA Press, pp: 62-65. 2015.

[3] Sanders DA, Stott I, Robinson D and Ndzi D. Analysis of successes and failures with a tele-operated mobile robot in various modes of operation. Robotica 30, pp 973-988. 2012.

[4] D Chikura, M Takahashi, S Watanabe and M Kitamura, Adaptation of User Behavior to the Different Level of Tele-Operation Support:. IEEE International Conference on Systems, Man, and Cybernetics. Volume 3, pp: 739 - 744. 1999.

[5] Backes PG, "Supervised Autonomy for Space Robotics," Progress in Astronautics and Aeronautics 161, pp. 139-158. I994

[6] Draper JV et.al. "Measuring Operator Skill and Teleoprator Performance," Proc. of International Symposium on Teleoperation and Contro1, 1998.

[7] Volpe R, "Techniques for Collision Prevention ,Impact Stability, and Force Control by Space Robots", Progress in Astronautics and Aeronautics, Vol.161, 1994, pp.I75-212.

[8] Gegov A, Arabikhan F, Sanders D, Vatchova B and Vasileva, T "Fuzzy networks with feedback rule bases for complex systems modelling". International Journal of Knowledge-Based and Intelligent Engineering Systems. 2017. In press.

[9] Gegov A, Petrov, N, Sanders D and Vatchova B, "Boolean matrix equations for node identification in fuzzy rule based networks", International Journal of Knowledge-Based and Intelligent Engineering Systems. 21, 2, p. 69-83. 2017.

[10] Gegov A, Petrov, N, Sanders D and Vatchova, B. "Modular rule base fuzzy networks for linguistic composition based modelling", International Journal of Knowledge-Based and Intelligent Engineering Systems. 21, 2, p. 53-67. 2017.

[11] Gegov A, Sanders D and Vatchova, B "Aggregation of inconsistent rules for fuzzy rule base simplification", International Journal of KnowledgeBased and Intelligent Engineering Systems. 2017. In press.

[12] Sanders D, Sanders B., Ndzi D and Bausch N, "Using confidence factors to share control between a mobile robot tele-operater and ultrasonic sensors", 2017 IEEE Proceedings Intelligent Systems Conference. In press.

[13] Sanders D, "Using self-reliance factors to decide how to share control between human powered wheelchair drivers and ultrasonic sensors. IEEE Transactions on Neural Systems and Rehabilitation Engineering", DOI: 10.1109/TNSRE.2016.2620988. Published by the IEEE.

[14] Sanders D, "Non-model-based control of a wheeled vehicle pulling two trailers to provide early powered mobility and driving experiences", IEEE Transactions on Neural Systems and Rehabilitation Engineering. DOI: 10.1109/TNSRE.2017.2726443. Published by the IEEE.

[15] Sanders D, Sanders B, Gegov A and Ndzi D. "Using confidence factors to share control between a mobile robot tele-operater and ultrasonic sensors", Intelligent Systems Conference 2017, 7-8 September 2017 | London, UK.

[16] Sanders D, Gegov A, Tewksbury G and Khusainov R. "Rule-based system to assist a powered wheelchair driver", Intelligent Systems Conference 2017, 7-8 September 2017 | London, UK.

[17] Sanders D, Gegov A, Tewksbury G and Khusainov R. "Rule-based system to assist a powered wheelchair driver", Intelligent Systems Conference 2017, 7-8 September 2017 | London, UK. 